\title{
FEDSM2008-55103
}

\section{CFD MODELING OF SLURRY FLOWS IN HORIZONTAL PIPES}

\author{
Franz H. Hernández \\ Simón Bolívar University \\ Department of Mechanics \\ Caracas, Venezuela \\ 02-35031@usb.ve
}

\author{
Armando J. Blanco \\ Simón Bolívar University \\ Department of Mechanics \\ Caracas, Venezuela \\ ajblanco@usb.ve
}

\author{
Luis Rojas-Solórzano \\ Simón Bolívar University \\ Department of Energy Conversion \\ Caracas, Venezuela \\ rrojas@usb.ve
}

\begin{abstract}
Liquid-solid two-phase flows are found in numerous operations in the chemical, petroleum, pharmaceutical and many other industries. In numerous cases, the mixture or slurry that flows is composed by a suspension of solid particles (dispersed phase) transported by a liquid (continuum phase). However, the large number and range of variables encountered in slurry flows, in the case of pipelines, cause the flow behavior of these slurry systems to vary over a wide range of conditions, and consequently, different approaches have been used to describe the behavior of different flow regimes. Therefore, there are numerous studies of particular cases that cover limited ranges of conditions. In consequence, the experimental approach is necessarily limited by geometric and physical scale factors. For these reasons, Computational Fluid Dynamics, CFD, constitutes an ideal technique for predicting the general flow behavior of these systems. CFD models in this area can be divided in two different classes: Eulerian-Eulerian and Lagrangian-Eulerian models. Differences between these models are related to the way the solid phase flow is represented. Lagrangian-Eulerian models calculate the path and motion of each particle, while EulerianEulerian models treat the particle phase as a continuum and average out motion on the scale of individual particles.
\end{abstract}

This work focuses on the Eulerian-Eulerian approach for modeling the flow of a mixture of sand particles and water in a horizontal pipe. Homogeneous and heterogeneous flow regimes are considered. The $\mathrm{k}-\varepsilon$ model was used for modeling turbulent effects. Additionally, closure of solid-phase momentum equations requires a description for the solidphase stress. Constitutive relations for the solid-phase stress considering the inelastic nature of particle collisions based on the Gas Kinetic Theory concepts have been used.
Governing equations are solved numerically using the control volume-based finite element method. An unstructured nonuniform grid was chosen to discretize the entire computational domain. A second-order scheme in space and time was used. Numerical solutions in fully developed turbulent flow were found.

Results show that flow predictions are very sensitive to the restitution coefficient and pseudo-viscosity of the solid phase. The mean pressure gradients from numerical solutions were compared with results obtained using the correlations of Einstein, Thomas and Krieger for homogeneous cases and with experimental data found in the open literature for heterogeneous cases. The solutions were found to be in good agreement with both correlations and experimental data. In addition, these numerical results were closer to experimental data than results obtained using other numerical models.

\section{NOMENCLATURE}

$\rho$

$\alpha$

$\overline{\bar{\tau}}$

$\overline{\bar{\tau}}$

$\mu$

$\lambda$

$\beta$

$C_{D}$

$d_{s}$

$\mathrm{Re}_{s} \quad$ Particle Reynolds Number

$P_{s} \quad$ Solid Pressure

$\Theta \quad$ Granular Temperature 


$\begin{array}{ll}e & \text { Coefficient of Restitution } \\ g_{0} & \text { Radial distribution function } \\ \gamma_{s} & \text { Particle collisions dissipation } \\ \alpha_{s, \max } & \text { Solid maximum packing concentration } \\ \lambda_{s} & \text { Solid bulk viscosity } \\ \varepsilon & \text { Dissipation of turbulent kinetic energy } \\ k & \text { Turbulent kinetic energy } \\ \mathrm{C}_{\mu}, \mathrm{C}_{\varepsilon}, \sigma_{\mathrm{k}}, \sigma_{\varepsilon} \text { Constants of the k- } \varepsilon \text { model } \\ \mathrm{P} & \text { Turbulent kinetic energy production } \\ \mu_{r} & \text { Relative shear viscosity }\end{array}$

\section{INTRODUCTION}

A slurry is a suspension of solid particles in a carrier liquid. Transport of slurries through circular pipelines is present in an important number of flows like in chemical, petroleum, water, pharmaceutical and other industries; and therefore, they have been the focus of much research. For example, in oil industry, efficient transport of rock cuttings, also named rubbles, generated at the bottom of a well is a major challenge when a long horizontal section has to be drilled. Drilling operations could be seriously limited if cuttings transport remains a problem in a hole. In particular, because of excessive drag and torque caused by small cuttings settled at the lower side of the horizontal well section, it may not be possible to introduce the casing in the hole. For these reasons, cuttings transport has been a major concern for years in the drilling industry. An investigation by Amoco [1] showed that $70 \%$ of lost time was due to unscheduled events associated with stuck pipe, while Hopkins \& Leicksenring [2] showed that a third of all stuck pipe problems are due to insufficient well bore cleaning. Thus, improving predictions of hydraulic efficiency of cuttings transport in horizontal drilling is very important in order to significantly alleviate costly problems and therefore, to maximize cost savings.

The most typical industrial case corresponds to slurries flowing in a single pipe. But, even this case is far from being simple. Complications appear because there is a large number of variables in slurry flows in pipelines: pipeline orientation, geometric shape of the conduit, roughness of conduit, particle size, particle size distribution, particle density, particle shape, local solid concentration, fluid density, fluid rheology and the properties of the fluid-particle interface.

In addition, several slurry flow regimes, or slurry flow patterns, could appear depending on the aforementioned variables and flow parameters such as mean flow velocity. A number of classifications of flow regimes have been suggested over the years [3-7]. One of the most common classifications are reported by $\mathrm{Hu}$ [8] who describes four different regimes: homogeneous, heterogeneous, heterogeneous with a moving bed and heterogeneous with a stationary bed. Homogeneous slurry flow shows an uniform distribution of solid particles across the pipeline transversal section. Heterogeneous slurry flow presents a solid concentration gradient in the vertical direction. Regimes with a bed appear when turbulence at the body of fluid is unable to support all particles in suspension and then, particle settling is present. When particles at the pipe bottom are able to move due to shear stress from fluid, bed is considered as a moving bed. However, when solid concentration at the bed reaches high values, close to maximum packing fraction, the bed becomes stationary.

Due to the wide range of possible values for different variables, there are numerous studies restricted to particular cases that cover a limited range of conditions [8]. Thus, experimental facilities are built for evaluating particular applications or for scaling field situations. In consequence, extrapolations of results are limited to cases very similar to those considered. On the other hand, it is very difficult to consider all variables and flow regimes for establishing theoretical models, even for particular cases.

For all these reasons, the numerical approach seems appropriated for modelling slurry flows. In recent years Computational Fluid Dynamics (CFD) has become a promising technique for multiphase flow modelling. In general, two different categories of CFD models are used for modelling two-phase flow. The Eulerian-Lagrangian model solves equations of motion for each individual particle, considering particle-particle collisions and the forces acting on each individual particle, whereas Eulerian-Eulerian models solve equations of motion for each phase considering both of them as fully interpenetrating continua.

Eulerian-Lagrangian models are impractical when the number of particles is appreciable. In this case, the computational effort required to obtain numerical solutions can be prohibitive. For this reason, Eulerian-Eulerian models are becoming most popular.

For liquid-solid mixtures, the Eulerian-Eulerian models may consider a granular temperature, in analogy as the Gas Kinetic Theory's temperature, and therefore, an energy balance equation is usually included within the set of governing equations. Different assumptions with respect to several aspects, such as: boundary conditions, interphase momentum transfer relationships, coefficients of restitution and radial distribution function, have been used. Turbulent flow is usually modelled with classical models such as $\mathrm{k}-\varepsilon$ or RNG k- $\varepsilon$.

Recently, some CFD's applications have been developed in the field of flows of fluidized beds [9] and liquid-solid slurry flows in a fully developed turbulent flow [10]. Cornelissen et al. [9] consider a two-dimensional EulerianEulerian approach and utilizes a commercial software package (ANSYS-Fluent) in vertical reactors. Ling et al. [10] consider 
slurry flows in horizontal pipes. The key aspect in their model is the way the slip velocity between phases is obtained. A simplified 3D algebraic slip mixture (ASM) model is introduced to obtain the numerical solution. Their ASM model can model two-phase flow (fluid or particulate) by solving the momentum equation and continuity equation for the mixture, the volume fraction equation for the secondary phase, and an algebraic expression for the relative (slip) velocity. Turbulence was considered by using the RNG $\mathrm{K}-\varepsilon$ turbulent model.

In this study, a full three-dimensional Eulerian-Eulerian model is used to model slurry flows. The slurry is a mixture of sand and water. Governing equations for each phase are solved numerically using the control volume-based finite element method. Turbulence was considered by using the $\mathrm{k}-\varepsilon$ model. A commercial software package (ANSYS-CFX ${ }^{\mathrm{TM}}$ ) is used to obtain the numerical solution.. Homogeneous and heterogeneous regimes without bed were considered. Sensitivity of flow predictions to restitution coefficient and pseudo-viscosity of the solid phase are analyzed. Pressure gradients predictions were compared with correlations proposed by Einstein [11], Thomas [12] and Krieger [13] for homogenous slurries. For heterogeneous regimes, model's predictions were compared with experimental data $[3,13]$ and numerical predictions from the ASM model [10].

In this paper, the governing equations are first presented, followed by the numerical scheme. Then, a validation process that assures convergence to numerical solutions is presented. Finally, relevant comparisons with correlations, experimental data and results from other numerical models let appreciate quality of numerical results obtained from the proposed model. Concluding remarks end the discussion.

\section{MATHEMATICAL MODEL}

The continuity equation for each phase is given by

$$
\left(\frac{\partial\left(\rho_{q} \alpha_{q}\right)}{\partial t}+\nabla\left(\rho_{q} \alpha_{q} \bar{V}_{q}\right)\right)=0
$$

where the subscript q represents either the solid or liquid phase, $\alpha$ the volume fraction, $\rho$ the density, $\bar{V}$ the velocity, and $t$ the time. Momentum equations for each phase are

$$
\begin{aligned}
& \rho_{l} \alpha_{l}\left(\frac{\partial \bar{V}_{l}}{\partial t}+\bar{V}_{l} \cdot \nabla \overline{V_{l}}\right)=-\alpha_{l} \nabla P+\nabla \cdot \alpha_{l} \overline{\overline{\tau_{l}}}+\alpha_{l} \rho_{l} \bar{g}-\beta\left(\overline{V_{l}}-\overline{V_{s}}\right) \\
& \rho_{s} \alpha_{s}\left(\frac{\partial \bar{V}_{s}}{\partial t}+\bar{V}_{s} \cdot \nabla \overline{V_{s}}\right)=-\alpha_{s} \nabla P+\nabla \cdot \overline{\bar{\tau}_{s}}-\nabla P_{s}+\alpha_{s} \rho_{s} \bar{g}+\beta\left(\overline{V_{l}}-\overline{V_{s}}\right)
\end{aligned}
$$

where the subscripts $l$ and $s$ represent the liquid and solid phase respectively, $\mathrm{P}$ the pressure, $\tau$ the stress tensor, $\mathrm{g}$ is the gravitational acceleration and $\beta$ the interphase exchange coefficient. Mass transfer between the phases is ignored while that lift and virtual mass forces are assumed to be negligible in the momentum equations. The stress tensor in equations (2) and (3) is

$$
\overline{\bar{\tau}}_{q}=\alpha_{q} \mu_{q}\left(\nabla \bar{V}_{q}+\nabla \bar{V}_{q}^{T}\right)+\alpha_{q}\left(\lambda_{q}-\frac{2}{3} \mu_{q}\right) \nabla \bullet \bar{V}_{q} \overline{\bar{I}}
$$

where $\lambda$ is the bulk viscosity, $\mu$ the shear viscosity and $I$ is the identity tensor. The interphase exchange coefficient in equations (2-3) is proposed by Gidaspow et al. [15] and includes expressions proposed by Wen and $\mathrm{Yu}$ [16] and Ergun [17]. It is expressed as

$$
\beta= \begin{cases}\frac{3}{4} C_{D} \frac{\alpha_{s} \alpha_{l} \rho_{l}\left|\bar{V}_{s}-\bar{V}_{l}\right|}{d_{s}} \alpha_{l}^{-2.65} & \alpha_{1}>0.8 \\ 150 C_{D} \frac{\alpha_{s}\left(1-\alpha_{l}\right) \mu_{l}}{\alpha_{l} d_{s}^{2}}+1.75 \frac{\rho_{l} \alpha_{s}\left|\bar{V}_{s}-\bar{V}_{l}\right|}{d_{s}} & \alpha_{1} \leq 0.8\end{cases}
$$

where, $d_{s}$ is the diameter of solid particles and $C_{D}$ is the drag coefficient, definition based on the particle Reynolds number as

$$
\begin{aligned}
& C_{D}=\frac{24}{\alpha_{l} \operatorname{Re}_{s}}\left[1+0.15\left(\alpha_{l} \operatorname{Re}_{s}\right)^{0.687}\right] \\
& \operatorname{Re}_{s}=\frac{\rho_{l} d_{s}\left|\bar{V}_{s}-\bar{V}_{l}\right|}{\mu_{l}}
\end{aligned}
$$

The solid pressure in equation (3) is obtained from Lun et al. [18]

$$
P_{s}=\rho_{s} \alpha_{s} \Theta\left[1+2(1+e) g_{0} \alpha_{s}\right]
$$

where $\Theta$ is the granular temperature, $e$ the restitution coefficient and $g_{0}$ the radial distribution function. The granular temperature is a measure of particle velocity fluctuations and it is obtained by the balance of granular energy. If only generation and dissipation terms are retained, the final expression for the balance is given by Lun et al.[18]

$$
0=\left(-\nabla P_{s} \overline{\bar{I}}+\overline{\bar{\tau}}_{s}\right): \nabla \bar{V}_{s}-\gamma_{s}
$$

with

$$
\gamma_{s}=\frac{12\left(1-e^{2}\right) g_{0}}{d_{s} \sqrt{\pi}} \rho_{s} \alpha_{s}^{2} \Theta^{3 / 2}
$$

where $\gamma_{s}$ represents the dissipation due to inelastic particleparticle collisions. In equations (8) and (10) it is necessary to include the radial distribution function. In this work, we tested two different expressions, proposed by Gidaspow et al.[15]

$$
g_{0}=\frac{3}{5}\left[1-\left(\frac{\alpha_{s}}{\alpha_{s, \max }}\right)^{1 / 3}\right]^{-1}
$$

and proposed by Lun et al. [18]

$$
g_{0}=\left(1-\frac{\alpha_{s}}{\alpha_{s, \max }}\right)^{-2.5 \alpha_{s, \max }}
$$


where $\alpha_{s, \max }$ is the maximum packing concentration. From these two expressions, Gidaspow et al.'s was adopted since it provided a better fitting.

The solid phase stress tensor for the solid phase requires of the bulk and shear viscosities. In the literature, there is general acceptation [18] on the form of the solid bulk viscosity proposed by Lun et al. [18]

$$
\lambda_{s}=\frac{4}{3} \alpha_{s}^{2} \rho_{s} d_{s} g_{0}(1+e) \sqrt{\frac{\Theta}{\pi}}
$$

However, there is not the same agreement regarding the solid shear viscosity. Van Wachen et al. [19] concluded that the choice of solid stress models do not have any significant impact on the results. In this work, we only considered the kinetic contribution of the shear viscosity that is given by

$$
\mu_{s}=\frac{5 \sqrt{\pi}}{48} \frac{\rho_{s} d_{p}}{(1+e) g_{0}}\left(1+\frac{4}{5}(1+e) g_{0} \alpha_{s}\right)^{2} \sqrt{\Theta}
$$

Since that slurry transportation is in the fully developed turbulent flow, a model for considering turbulence must be used. In this work the k- $\varepsilon$ model was used. The turbulent kinetic energy equation in $\mathrm{k}-\varepsilon$ model is

$$
\frac{\partial(\rho \bar{V} \otimes \bar{V})}{\partial t}+\nabla \cdot(\rho \bar{U} \otimes \bar{V} \otimes V)=P+\varphi+\nabla \cdot\left(\left(\mu+\frac{2}{3} c_{s} \rho \frac{\kappa^{2}}{\varepsilon}\right) \nabla \bar{V} \otimes \bar{V}-\frac{2}{3} \delta \rho \varepsilon\right)^{(15)}
$$

while that dissipation rate of turbulent kinetic energy is obtained from

$$
\frac{\partial(\rho \varepsilon)}{\partial t}+\nabla \bullet(\rho U \varepsilon)=\frac{\varepsilon}{k}\left(c_{\varepsilon 1} P-c_{\varepsilon 2} \rho \varepsilon\right)+\nabla \cdot\left(\left(\mu+\frac{\mu_{t}}{\sigma_{\varepsilon}}\right) \nabla \varepsilon\right)
$$

And the Prandtl-Kolmogorov expression

$\mu_{T}=C_{\mu} \rho \frac{k^{2}}{\varepsilon}$

With the customary constant coefficients

$$
\begin{aligned}
& \mathrm{C}_{\mu}=0.09 \\
& \mathrm{C}_{\varepsilon 1}=1.44 \\
& \mathrm{C}_{\varepsilon 2}=1.92 \\
& \sigma_{\mathrm{k}}=1 \\
& \sigma_{\varepsilon}=0.44
\end{aligned}
$$

In practical situations, when the slurry flow is considered homogeneous, the system of fluids is represented as an homogeneous mixture of solid and liquid phases. In order to establish limits of the validity of some correlations under such a condition, flows of fluid mixtures were considered. For these mixtures the density is expressed as

$$
\rho_{m}=\alpha_{s} \rho_{s}+\left(1-\alpha_{s}\right) \rho_{l}
$$

while its viscosity is expressed as

$$
\mu_{m}=\mu_{r} \mu_{l}
$$

where $\mu_{r}$, the relative viscosity, is expressed following Einstein's correlation [11]

$$
\mu_{r}=1+2.5 \alpha_{s}
$$

Thomas's correlation [12]

$$
\mu_{r}=1+2.5 \alpha_{s}+10.05 \alpha_{s}^{2}+0.00273 e^{16.6 \alpha_{s}}
$$

or Krieger's correlation [13]

$$
\mu_{r}=1-\frac{\alpha_{s}}{\alpha_{s, \max }}
$$

where the subscript $\mathrm{m}$ represents the mixture properties.

\section{NUMERICAL MODEL}

All governing equations of slurry flows, with appropriated boundary conditions, were solved in a Cartesian coordinate system by using the CFD commercial software ANSYS$\mathrm{CFX}^{\mathrm{TM}}$. Heat transfer was neglected and steady state was considered in all cases. The slurry properties came from a mixture of water and silica sand.

In order to validate the numerical model, the geometry and physical problem used in this work are the same assumed by Ling et al. [10]. So, comparisons with numerical results and experimental data generated by Skudarnov el al. [14] and Newitt et al. [3] were possible. In consequence, the geometrical domain is considered as an horizontal straight pipeline of length $\mathrm{L}=3 \mathrm{~m}$ and diameter $\mathrm{d}=0.0221 \mathrm{~m}$. Pipe length was chosen in order to assure that a steady fully developed flow is well reached before the end of the annulus. This lets a zone, whose velocity profile is independent of the position along the pipe axis. Thus, any effect of boundaries (entrance or outlet) on the results are avoided.

The range of solid volume fraction was $0-10 \%$ for validation of correlations for pseudo-homogeneous mixtures. Only $20 \%$ of volume fraction of solid was considered for heterogeneous cases.

A multi-block unstructured non-uniform grid system with hexahedral elements was used to discretize the entire computational domain.

For the grid construction, mesh seeds were located in the axial, circular, and radial direction of the models, and then, hexahedral elements were generated. Hexahedral elements allow better results than tetrahedral ones in terms of computing time and accuracy [20]; therefore, the former types of elements are selected for this study. A typical grid is shown in figure 1. 


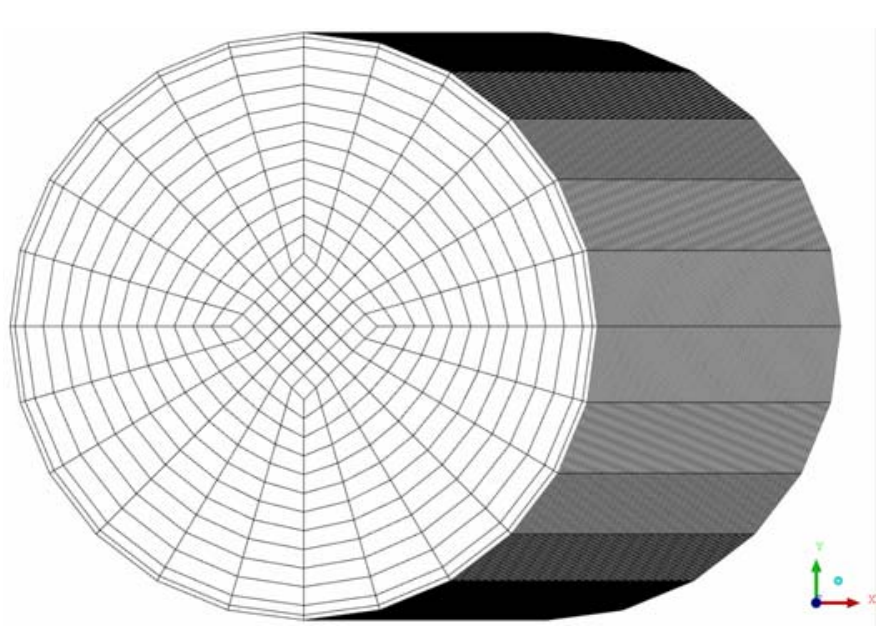

Figure 1. Typical grid for the horizontal pipeline domain.

In order to select the appropriate grid element density for the entire flow condition of interest, several grids were considered. Values of velocities and pressure gradients along the pipe axis were compared between different grids. A grid was considered adequate once the average velocities and pressure gradients, evaluated at the fully developed flow zone, kept a relative difference lesser than 3\% when compared with the results obtained by using a refined grid with a doubled number of elements. For establishing radial, circumferential and longitudinal element density, pipeline length was fixed in $\mathrm{L}=1 \mathrm{~m}$. Refinement was done simultaneously in the radial, circular and longitudinal directions. For grid tests, particle diameter was $30 \mu \mathrm{m}$, particle density $2390 \mathrm{Kg} / \mathrm{m}^{3}$, solid shear viscosity $0.0001 \mathrm{~Pa}-\mathrm{s}$, coefficient of restitution 0.9 , maximum packing concentration 0.62 , volume fraction of solids $5 \%$, mixture velocity at the entrance of $3 \mathrm{~m} / \mathrm{s}$ (mass flow 0.765 $\mathrm{kg} / \mathrm{s}$ ). Pipe roughness was $50 \mu \mathrm{m}$.

Three different combination of radial-longitudinal number of elements were considered: $6 \times 20,12 \times 40$ and $25 \times 80$. Values of axial pressure gradients and velocities are shown in figure 2 .
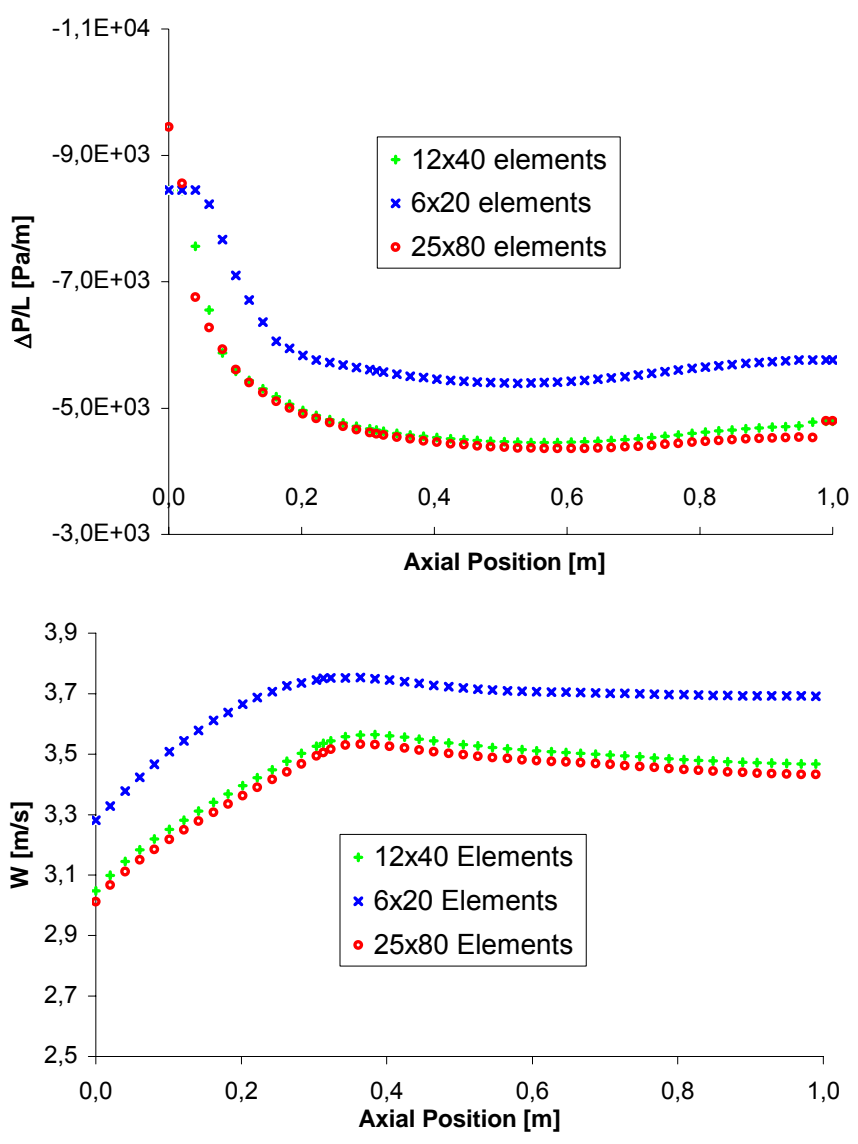

Figure 2. Comparison of numerical predictions of axial pressure gradient and velocities for grid selection.

These results keep a difference lesser than $2.5 \%$ between $12 \times 40$ and $25 \times 80$ grids which suggests that reliable numerical convergence is achieved with the $12 \times 40$ grid. A $16 \times 50$ grid was used.

Conditions imposed at each boundary are: (a) Inlet: the same mean velocity for each phase and uniform solid distribution, (b) Outlet: pressure reference value, (c) Wall: non-slip condition.

Pseudo-time discretization is done with the second order backward-difference scheme. Solution of the pressure field requires a pressure correction equation, correcting the pressure and velocities after each iteration for verifying continuity equations; for this, the well known algorithm SIMPLE is employed.

Convergence criteria of $10^{-4}$ and $10^{-6}$ for each scaled residual component (scaled residual of the linear momentum in each direction for each phase) were employed. Results showed to be very sensitive to mass conservation criteria.

Sensitivity of results to solid viscosity value was analyzed. It was found that for solid viscosity values lower than 0.0001 
Pa-s results were independent of this value. Therefore, this value was used in all cases here analyzed.

\section{RESULTS}

In this work, homogeneous and heterogeneous flow regimes without any kind of bed were considered. In the case of homogeneous flow, comparisons between predictions of pressure gradients at the fully developed zone were done with those obtained considering the flow of an equivalent homogeneous fluid (EHF). Density of EHF was obtained by using equation (17), while that viscosity was computed through the equations (18-21) depending on which correlation was considered (Einstein, EFHE, Thomas, EFHT, or Krieger, EFHK).

Sensitivity of multiphase flow simulations to solid viscosity values was analyzed. The solid viscosity value was adjusted in order to satisfy a pressure gradient value obtained from EHFE for a particular value of solid concentration $(0.75 \%)$. Hence, a value of $0.00317 \mathrm{~Pa}-\mathrm{s}$ was prescribed for the solid viscosity. Then, this value was used in all pseudohomogeneous cases of solid concentration analyzed.

Figure 3 shows variation of pressure gradients for different solid concentration values. The maximum difference in the range considered was lower than $0.5 \%$ (at higher solid concentration values).

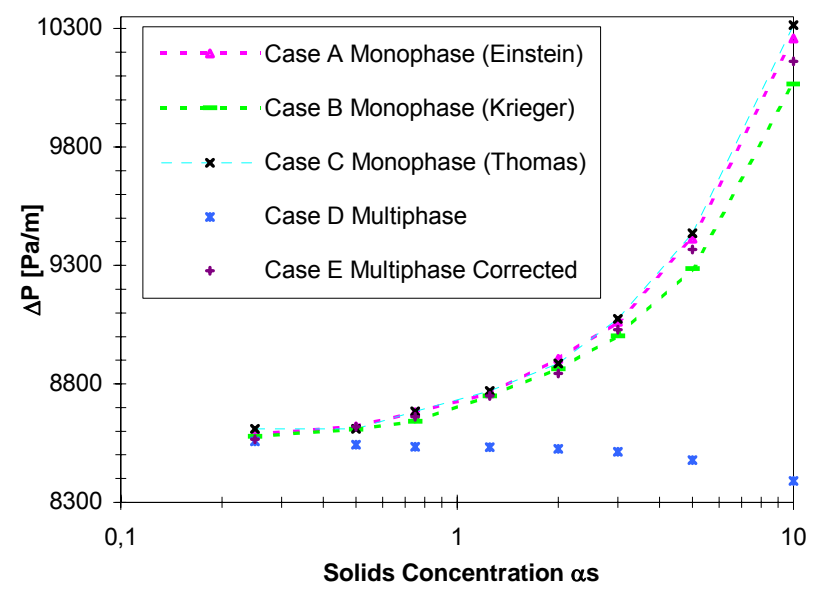

Figure 3. Comparison of pressure gradient predictions for (a) EHF Einstein, (b) EHF Thomas (c) EHF Krieger, (d) slurry flows and (e) slurry flows with fixed solid viscosity

The huge range of values considered for solid concentration and the ample variations in pressure gradient predictions $(20 \%)$ permit to conclude that the value assigned to solid viscosity leads to accurate simulations of this kind of flow.

However, other studies that consider different values of flow parameters and pipe geometry should be done before establishing conclusions about the role of the solid viscosity value.

In particular, it should be established the value of solid viscosity as an universal constant or its dependency with flow parameters for modelling homogeneous slurry flows.

The second flow regime here considered, is related with heterogeneous flow. To verify and check the numerical results from the proposed model, comparison with experimental results obtained for silica sand-water slurries by Skudarnov et al. [14] and Newitt et al. [3] and with numerical results from ASM model proposed by Ling et al. [10] were accounted for.

Parameters of heterogeneous flow are shown in table 1.

Table 1 Geometrical and physical properties for heterogeneous simulations.

\begin{tabular}{|c|c|c|c|c|c|}
\hline $\begin{array}{c}\mathrm{D}_{\mathrm{i}} \\
(\mathrm{cm})\end{array}$ & $\begin{array}{c}\mathrm{L} \\
(\mathrm{cm})\end{array}$ & $\begin{array}{c}\rho_{\mathrm{L}} \\
\left(\mathrm{Kg} / \mathrm{m}^{3}\right)\end{array}$ & $\begin{array}{c}\rho_{\mathrm{S}} \\
\left(\mathrm{Kg} / \mathrm{m}^{3}\right)\end{array}$ & $\begin{array}{c}\mu_{\mathrm{L}} \\
(\mathrm{cP})\end{array}$ & $\begin{array}{c}\mu_{\mathrm{S}} \\
(\mathrm{cP})\end{array}$ \\
\hline 2.21 & 140 & 998.2 & 2381 & 0.89 & 0.01 \\
\hline
\end{tabular}

The solid concentration was set in $20 \%$. Sensitivity of pressure gradient to solid viscosity value was analyzed. Also, the effect of considering kinetic theory for taking into account the interaction between solid particles was studied.

For this geometry, Skudarnov et al. [14] determined that critical velocity is close to $0.97 \mathrm{~m} / \mathrm{s}$. Therefore, mixture velocities considered were in the range of $1 \mathrm{~m} / \mathrm{s}$ to $2.75 \mathrm{~m} / \mathrm{s}$ according with experimental data.

In heterogeneous flows, it was found no influence in reducing solid viscosity onto predictions of pressure gradient for the range of mixture mean velocity analyzed. Solid viscosity was decreased in four orders of magnitude and the mean pressure gradient difference was less than $1.5 \%$.

Influence of considering kinetic theory for simulating pressure gradient was estimated. Figure 4 shows pressure gradient predictions with and without using kinetic theory.

The difference between predictions using kinetic theory and mean experimental results (considering both Skudarnov and Newitt's results) was estimated in $9.5 \%$ and $9.8 \%$ respectively. Additionally, the mean difference without using kinetic theory was estimated in $10.4 \%$ and $9.8 \%$, respectively. However, both predictions were closer to experimental data than predictions from the ASM model (16\%). 


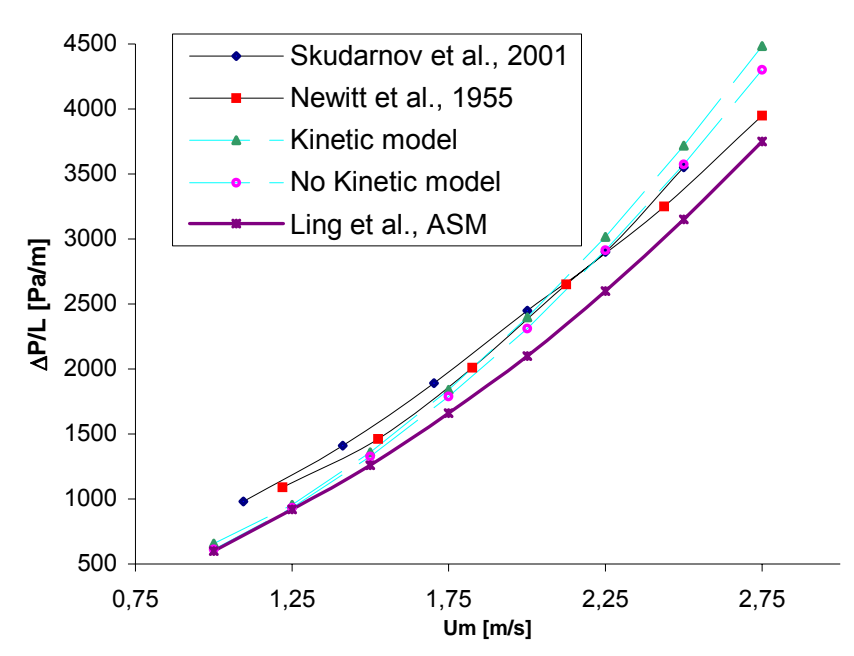

Figure 4. Comparison of pressure gradient predictions between numerical results, ASM model and experimental data from for Skudarnov et al. and Newitt et al.

For lower values of mean slurry velocity, predictions of all numerical models are equals. When mean velocity is increased, full multiphase simulation perform better than ASM model. In addition, the use of kinetic theory slightly improves pressure gradient predictions when the velocity is reduced in comparison with modelling without kinetic theory. This is due to the estimation of particle interactions. However, a difference in solid concentration distribution is observed when kinetic theory is not employed.

\section{CONCLUSIONS}

CFD modelling of homogeneous and heterogeneous slurry flows shows be a technique that lets obtain good estimation of important flow parameters as mean pressure gradient.

Full multiphase flow simulations provide excellent results for homogeneous slurry flows. In these cases, solid viscosity must be estimated in order to produce numerical predictions in agreement with numerical correlations. However, more research should be done in order to determine adequate values for solid viscosity.

Full multiphase flow simulations provide better predictions than the ASM model for the heterogeneous liquidsolid slurry. In addition, the numerical predictions for the pressure gradients are in good agreement with the experimental data.

Including kinetic theory for taking into account particle interaction improves the results compared to those obtained when kinetic theory is not considered. However, for high values of the mixture velocity, predictions of pressure gradients are not as good as they are for medium values. This point will be objet of new research.

\section{ACKNOWLEDGMENTS}

The author wishes to acknowledge to the FONACIT, Venezuela, for supporting this project.

\section{REFERENCES}

1. Massie G. W., Castle-Smith J., Lee J.W. and Ramsey M. S. 1995, Amoco's Training Iniciative Reduce Wellsite Drilling Problems, Petroleum Engineer International

2. Hopkins C. J. \& Leicksenring R. A., 1995, Reducing the risk of Stuck Pipe in the Netherlands, IADC/SPE 29422.

3. Newitt, D. M., Richardson J. F., Abbot M. \& Turtle R. B. 1955, Hydraulic conveying of solids in horizontal pipes. Trans. Inst. Chem. Eng. 33, 93-113.

4. Thomas D. G. 1964, Transport characteristics of suspensions, Part. IX, AIChE J., 10, 303-308.

5. Wicks M., Transportation of solids of low concentrations in horizontal pipes. 1971, Advances Solid-Liquid Flow in Pipes and Its Application, Zandi, I., Ed., Pergamon Press, New York.

6. Ercolani, D., Ferrini, F., and Arrigoni, V. 1979, Electric and thermic probes for measuring the limit velocity, Proceedings of the Hydrotransport 6 Conference, BHRA Fluid Eng., Cranfield, U.K., Paper A3, pp. 27-42.

7. Brown, N.P. 1991, Flow regimes of settling slurries in pipes, Slurry Handling Design of Solid-Liquid Systems, Brown, N.P. and Heywood, N.L., Eds., Elsevier, London, pp. 41-52.

8. Hu S. 2006, Slurry Flows, Multiphase Flow Handbook, Chapter 4, Clayton T. Crowe Ed., Taylor \& Francis Group, Boca Raton, pp. 50-101

9. Cornelissen J. T., Taghipour F., Escudié R., Ellis N, \& Grace J. R. 2007, CFD modelling of a liquid-solid fluidized bed, Chemical Engineering Science, 62, pp 63346348.

10. Ling J., Skudarnov P.V., Lin C.X., Ebadian M.A. 2003, Numerical investigations of liquid-solid slurry flows in a fully developed turbulent flow region, International Journal of Heat and Fluid Flow, 24, pp. 389-398

11. Einstein, A., 1906, Zur Theorie der Brownschen Bewegung, Annalen der Physik, 19, pp. 248-258.

12. Thomas, D.G. 1965, Transport characteristics of suspensions: VIII. A note on the viscosity of Newtonian suspensions of uniform spherical particles, J. Colloid Sci., 20, 267-277.

13. Krieger, I.M., Rheology of monodisperse lattices, Adv. Colloid Interface Sci., 3, 111-136, 1972.

14. Skudarnov, P.V., Kang, H.J., Lin, C.X., Ebadian, M.A., Gibbons, P.W., Erian, F.F., Rinker, M. 2001. Experimental Investigation of Single- and Double-Species Slurry Transportation in a Horizontal Pipeline. Proc. ANS 9th International Topical Meeting on Robotics and Remote Systems, Seattle, WA.

15. Gidaspow, D., Bezburuah, R., Ding, J., 1992. Hydrodynamics of circulating fluidized beds, kinetic 
theory approach. In: Potter, O.E., Nicklin, D.J. Eds., Fluidization VII. Engineering Foundation, New York, pp. 75-82.

16. Wen, C.Y., Yu, Y.H., 1966. Mechanics of fluidization. Chemical Engineering Progress Symposium Series 62, 100111.

17. Ergun, S., 1952. Fluid flow through packed columns. Chemical Engineering Progress 48, 89-94.

18. Lun, C.K.K., Savage, S.B., Jeffrey, D.J., Chepurniy, N., 1984. Kinetic theories for granular flow: inelastic particle in Couette flow and slightly inelastic particles in a general flow field. Journal of Fluid Mechanics 140, 223256.
19. van Wachem, B.G.M., Schouten, J.C., Bleek van den, C.M., Krishna, R., Sinclair, L.L., 2001. Comparative analysis of CFD models of dense gas-solid systems. A.I.Ch.E. Journal 47, 1035-1051.

20. Hernández F., 2006, BSc in Mechanical Engineering Dissertation Project. Universidad Simón Bolívar Venezuela. 